\title{
Interdisciplinarité, interdisciplinarités
}

Interdisciplinarity, interdisciplinarities

\section{Béatrice Fleury et Jacques Walter}

\section{OpenEdition}

Journals

Édition électronique

URL : http://journals.openedition.org/questionsdecommunication/409

DOI : 10.4000/questionsdecommunication.409

ISSN : 2259-8901

\section{Éditeur}

Presses universitaires de Lorraine

\section{Édition imprimée}

Date de publication : 1 décembre 2010

Pagination : 145-158

ISBN : 978-2-8143-0056-9

ISSN : 1633-5961

\section{Référence électronique}

Béatrice Fleury et Jacques Walter, «Interdisciplinarité, interdisciplinarités », Questions de communication [En ligne], 18| 2010, mis en ligne le 01 décembre 2012, consulté le 30 avril 2019. URL: http://journals.openedition.org/questionsdecommunication/409; DOI : 10.4000/ questionsdecommunication.409 


\section{$>$ ÉCHANGES}

BÉATRICE FLEURY

Centre de recherche sur les médiations

Université Nancy 2

Beatrice.Fleury@univ-nancy2.fr

JACQUES WALTER

Centre de recherche sur les médiations

Université Paul Verlaine-Metz

jacques.walter@univ-metz.fr

\section{INTERDISCIPLINARITÉ, INTERDISCIPLINARITÉS}

Résumé. - Patrick Charaudeau se définit sémiologue et chercheur en analyse du discours. C'est donc à ce titre qu'il intervient ici. En effet, sur un ton non polémique mais avec conviction, il définit ce qu'est une discipline pour penser dans quels cadre et conditions l'interdisciplinarité peut s'accomplir. Deux linguistes - Dominique Maingueneau et Roselyne Koren - et une chercheuse en sciences de l'information et de la communication - Sylvie Leleu-Merviel - lui répondent, chacun précisant ce que recouvre les notions de discipline et d'interdiscipline, les deux premiers en abondant - à quelques nuances près - dans le sens des propositions de Patrick Charaudeau, la dernière en les mettant à l'épreuve de la construction scientifique.

Mots clés. - Interdisciplinarité, interdisciplinarité focalisée, analyse du discours, sciences du langage, discipline, champ disciplinaire, phénomènes de communication. 
$\mathrm{D}$ ans la rubrique «Échanges 》 de Questions de communication, il est régulièrement débattu de sujets en lien avec des préoccupations épistémologiques : le constructivisme $(5,2004 a ; 6,2004 b)$ ), les problèmes posés par le terrain et l'expérimentation (7, 2005a; 8, 2005b), l'histoire des sciences de l'information et de la communication $(12,2007)$, la question du genre en sciences humaines et sociales $(15,2009 a ; 16,2009 b)$. Si le thème de l'interdisciplinarité pouvait se retrouver en arrière-fond des arguments développés pour chacun (plus particulièrement quand il s'est agi de traiter de l'histoire des sciences de l'information et de la communication), jamais encore il n'avait fait frontalement l'objet d'une interrogation. Pourquoi? Probablement parce que ce postulat est si présent en sciences de l'information et de la communication (SIC) qu'il peut donner à penser que, non seulement, il relève de l'évidence, mais que l'ensemble des chercheurs du secteur en maîtrisent les fondements et façons de faire. Ceci explique probablement aussi qu'il ait été difficile de convaincre des collègues d'accepter de collaborer à ces « Échanges ». Parmi les justifications données, figurait notamment celle selon laquelle le sujet avait déjà été débattu. Au vu de ceci, pourquoi traiter de l'interdisciplinarité alors que, aujourd'hui, elle est régulièrement convoquée au nom « de la complexité croissante du savoir » (Charaudeau, 2010 : 197)? C'est précisément parce qu'elle est si présente qu'il est intéressant de savoir comment elle est pratiquée. C'est d'ailleurs ce qu'énonce Patrick Charaudeau : «Ce n'est pas de cette façon polémique que j'aborderai ici la question de l'interdisciplinarité. Je prendrai plutôt le parti d'une interrogation sur la possible rencontre de disciplines. Mais si une telle rencontre s'impose, comment celle-ci doit-elle s'opérer? » (ibid.).

Pour répondre à cette question, Patrick Charaudeau se fonde sur sa propre identité de chercheur, celle d'un sémiologue et d'un analyste du discours. Et s'il le fait, c'est qu'il considère que, pour traiter des conditions de l'interdisciplinarité, il faut partir d'une interrogation générique sur ce qu'est une discipline, puis sur ce qui caractérise les phénomènes sociaux et leur problématisation. En découle la mise en exergue de notions et questionnements dont il apparait qu'ils sont partagés par différentes disciplines. Fort de ce cadrage, Patrick Charaudeau en vient à une position théorique et méthodologique - l'interdisciplinarité focalisée - dont il démontre la pertinence dans le cadre de l'étude de plusieurs domaines : l'identité du sujet, la représentation associée à celle d'imaginaire social, la stratégie.

Pour lui répondre: deux linguistes - Dominique Maingueneau et Roselyne Koren - et une chercheuse en sciences de l'information et de la communication, Sylvie Leleu-Merviel. Les linguistes abondent dans le sens de la démonstration de leur collègue, le premier s'intéressant plus particulièrement à la définition de ce qu'est un champ disciplinaire tandis que la seconde met en pratique les présupposés de ce qu'est une interdisciplinarité focalisée. Avec une réserve néanmoins pour Dominique Maingueneau qui préfère complexifier l'approche de Patrick Charaudeau en ce qui concerne la notion de champ disciplinaire. Quant 
à Sylvie Leleu-Merviel, elle déplace la focale, mettant à distance celle de l'analyse du discours qui permettait au linguiste de penser l'acte de communication et l'interdisciplinarité. En découle une autre façon de penser la science dans laquelle l'idée d'horizon d'attente du texte de départ.

Les deux questions principales qui sont au cœur de ces « Échanges » sont donc en phase avec celles posées par l'analyste du discours - «Qu'est-ce qu'une discipline? », « Que signifie pratiquer l'interdisciplinarité? ». En bonne logique, elles guideront notre propos.

\section{Qu'est-ce qu'une discipline?}

Dans Qu'est-ce qu'une discipline? (Boutier, Passeron, Revel, 2006), des chercheurs partent du constat de l'évolution des sciences pour interroger les liens aujourd'hui entretenus entre les institutions scientifiques et réfléchir dans le même temps à la question des frontières disciplinaires. D'ailleurs, dans l'introduction aux «Échanges 》 consacrés à l'histoire des sciences de l'information et de la communication, nous citions (Fleury, Walter, 2007: 134) un auteur de cet ouvrage, Jean-Louis Fabiani (2006: 12-13), qui expliquait qu'il convenait de se méfier de l'illusion naturaliste pour définir une discipline et précisait que la notion juxtapose deux ordres historiques de réalité en tension :

\footnotetext{
« Le premier est bien antérieur à la science moderne. II comprend toutes les dimensions d'un rapport pédagogique mais désigne primitivement la relation vue à partir de l'élève, du discipulus, c'est-à-dire sous l'aspect de la réception des messages. La discipline ainsi entendue met l'élève au centre du système et organise le savoir à partir d'un objectif pédagogique. Plus qu'un sujet connaissant, ce sont les formes du contrôle pédagogique qui occupent ici une place centrale. II s'agit de s'assurer de la réalité et de l'efficacité d'une autorité pédagogique en tant qu'elle s'exprime à travers une opération de communication. Le deuxième objet, central dans nos préoccupations depuis le XIX ${ }^{e}$ siècle, c'est l'organisation particulière au sein de laquelle se développent des savoirs modernes comme ensemble de pratiques codifiées et reconnues valides par un collectif auto-délimité, l'activité scientifique se développant par rapport à une ligne de front ».
}

Pourquoi reprendre cette définition? En fait, afin de la confronter à celle que propose Patrick Charaudeau qui retient plus particulièrement le deuxième aspect. Pour lui, en effet,

« une discipline est constituée d'un certain nombre de principes fondateurs, d'hypothèses générales, de concepts qui déterminent un champ d'étude et permettent en même temps de construire le phénomène en objet d'analyse. Se constitue ainsi un cadre conceptuel, et c'est à l'intérieur de celui-ci que peuvent être construites diverses théories [...]. Sans cadre théorique, point de discussion possible car on ne saurait dire au nom de quoi on pourrait évaluer, confirmer ou contester les résultats d'une analyse » (Charaudeau, $2010: 200-201$ ).

Et d'ajouter que c'est au vu de ce cadrage que d'aucuns dénient aux sciences de l'éducation ou aux sciences de l'information et de la communication « le 
label de discipline » (ibid.). Aussi suggère-t-il de qualifier ces secteurs de champs disciplinaires, une appellation « utile pour éviter les querelles » et qu'il convoque aussi pour la psychologie ou les sciences du langage. Les disciplines peuvent se combattre et se jauger au prisme des savoirs et connaissances qui les constituent. Or, c'est précisément sur ce point qu'intervient Dominique Maingueneau qui considère qu'un champ disciplinaire se caractérise lui aussi par son hétérogénéité et, donc, par un déséquilibre - et un jeu de pouvoir - entre sous-disciplines à l'intérieur de celui-ci. Ainsi évoque-t-il, au sein d'un même champ, l'existence de secteurs plus porteurs que d'autres, telle la psychologie cognitive aujourd'hui, avec laquelle il est de bon ton de s'associer, au-delà du principe d'interdisciplinarité.

« Bien entendu, un chercheur en analyse du discours ou en sociologie qui veut travailler avec des psychologues peut minorer la psychologie cognitive, mais s'il le fait, il aura de moins bonnes chances d'accéder à des financements et d'être reconnu dans son domaine. L'interdisciplinarité au sens fort est une nécessité pour toute recherche scientifique, puisque toute vraie innovation dans ce domaine implique que les chercheurs sortent de leur espace, qu'ils entrent en dialogue avec d'autres modèles, d'autres disciplines, d'autres façons de penser. Mais cette nécessité peut aboutir à des résultats très variés, qui peuvent aller dans des sens opposés ».

D'où le constat selon lequel le bornage relatif au savoir qui délimite les zones disciplinaires connaît des tensions et ajustements qui font « que leur survie n'est jamais assurée » et qu'« elles ne sortent pas nécessairement renforcées de toute mise en relation ». Dominique Maingueneau ajoute à cette complexité un autre facteur de brouillage :l'hétérogénéité des référents disciplinaires des sous-champs d'un même champ disciplinaire. Pour cela, il fonde sa démonstration sur l'analyse du discours. Partons de cette idée quelque peu simplificatrice mais utile pour comprendre le raisonnement de Dominique Maingueneau :un sous-champ décline un aspect des prérogatives d'un champ disciplinaire; il le fait en se spécialisant sur un type d'interrogations et un domaine d'intervention particulier. Pour autant, selon Patrick Charaudeau, l'ensemble des sous-champs se retrouverait sur des bases théoriques communes. II en serait ainsi de ceux de sciences du langage - la linguistique descriptive de la langue, la linguistique cognitive, la sociolinguistique, l'ethnolinguistique, la linguistique du discours - qui se réfèreraient tous - comme le résume Dominique Maingueneau - à « des concepts communs de signe, forme et sens, ordres syntagmatique et paradigmatique énoncé/énonciation et de procédures d recueil et analyse de corpus ». Mais, pour ce dernier, le problème viendrait de là. Selon lui, la juxtaposition tend à masquer l'hétérogénéité qui fait que chaque sous-champ ne se définit pas nécessairement en fonction des mêmes disciplines. Un exemple : la grammaire générative se définit comme une branche de la psychologie alors que, pour ses praticiens, « elle est apparue avant tout comme une approche qui permettait de modéliser des propriétés du système linguistique mieux que ne pouvaient le faire d'autres courants linguistiques. Les considérations d'ordre psychologique jouaient finalement un rôle marginal dans cette affaire ». Même brouillage quand il s'agit de raconter l'histoire de l'analyse du discours, les auteurs identifiés comme « ayant joué un rôle dans sa fondation $[\ldots]$ se trouvent placés dans des situations d'interdisciplinarité très diverses ». La 
conséquence est sans appel : « Autant dire que même l'inscription de l'analyse du discours dans l'orbite des sciences du langage ne peut aller de soi ».

Sur le plan de l'histoire d'une discipline en lien avec les concepts qui la fondent, les frontières sont donc difficiles à tracer. Et sur le plan des communautés? Pour Dominique Maingueneau, si ce versant « sociologique » n'est pas frontalement abordé par Patrick Charaudeau, il figure en arrière-fond de sa réflexion. Et de citer Jean-Marie Berthelot (1996: 99-100) pour qui « espace social de légitimation de savoirs, une discipline est, indissociablement, un espace de construction d'argumentations ». Versants épistémologique et sociologique sont en interrelations constantes, avec toutefois des variations selon les cas. De ce point de vue, Dominique Maingueneau note que Patrick Charaudeau n'a pas traité de la dimension institutionnelle de la construction des disciplines, probablement parce qu'il s'exprime depuis le domaine de l'analyse du discours qui, de fait, connaît un versant scientifique mais ne dépend que faiblement d'un versant institutionnel. Et pourtant... Une discipline est indéniablement formatée par un cadre organisationnel. Ce qui fait dire à Edgar Morin (1990) que les idées y font corps avec l'organisation qui les constitue :

\begin{abstract}
« La discipline est une catégorie organisationnelle au sein de la connaissance scientifique; elle y institue la division et la spécialisation du travail et elle répond à la diversité des domaines que recouvrent les sciences. Bien qu'englobée dans un ensemble scientifique plus vaste, une discipline tend naturellement à l'autonomie, par la délimitation de ses frontières, le langage qu'elle se constitue, les techniques qu'elle est amenée à élaborer ou à utiliser, et éventuellement par les théories qui lui sont propres. L'organisation disciplinaire s'est instituée au XIXe siècle, notamment avec la formation des universités modernes, puis s'est développée au $X X^{e}$ siècle avec l'essor de la recherche scientifique; c'est-à-dire que les disciplines ont une histoire: naissance, institutionnalisation, évolution, dépérissement etc.; cette histoire s'inscrit dans celle de l'université, qui, elle-même, s'inscrit dans l'histoire de la société; de ce fait les disciplines relèvent de la sociologie des sciences et de la sociologie de la connaissance et d'une réflexion interne sur elle même, mais aussi d'une connaissance externe. II ne suffit donc pas d'être à l'intérieur d'une discipline pour connaître tous les problèmes afférents à celle-ci ».
\end{abstract}

C'est d'ailleurs cette histoire, nourrie des pensées et actions de chercheurs au sein des organisations qu'ils ont contribué à façonner et transformer, que Robert Boure $(2006,2007,2008)$ a étudiée à propos des sciences de l'information et de la communication. Investir de la sorte cette discipline lui a permis de mettre notamment à l'épreuve l'idée selon laquelle l'origine de celle-ci serait littéraire et de montrer, exemples à l'appui - thèses soutenues, premiers lieux d'enseignement et de recherche en France, revues du champ - qu'elle est plurielle. Et si, à partir d'une focale à large portée - l'université française - Robert Boure est parvenu à retracer des expériences particulières, Stéphane Olivesi (2007 : 205) est quant à lui parti d'une perspective inversée pour montrer comment une histoire « localisée » peut elle aussi - voire surtout - être riche d'enseignements en bousculant la vision mythifiée qu'une histoire générale peut façonner:

« Cette thèse [...] se propose de déplacer les points de vue, d'opposer à une connaissance générale, élaborée à partir de sources nationales et officielles, des éléments de savoir locaux, éclatés, irréductibles à une vérité exclusive, bref d'opposer à une histoire froide (ou refroidie) 
des mémoires encore vivantes et souvent discordantes, potentiellement en lute dans la définition de la vérité historique. Cette histoire que l'on qualifiera volontiers de matérialiste suggère que la discipline n'existe pas par ses œuvres de l'esprit, par le subtil dessein de quelques visionnaires « Pères fondateurs », par la force du groupe institutionnel qui l'a fait émerger et la porte, mais par le développement local de lieux d'enseignement (plus que de recherche) ».

Que retenir de ceci? Avant tout :l'hétérogénéité. Pas seulement celle qui distingue les disciplines les unes des autres, mais celle qui est constitutive de leur existence et qui fait qu'elles sont composées d'histoires diverses et contrastées selon les lieux et spécialités. Et si l'existence même des disciplines est envisagée comme pouvant être une force car donnant un cadre et des repères aux chercheurs, elle peut aussi être un frein à l'ouverture de chacun à d'autres façons de penser. C'est d'ailleurs plutôt cet aspect qu'aborde Roselyne Koren pour qui l'interdisciplinarité représente un facteur de richesse, tandis que Sylvie Leleu-Merviel explique pourquoi ce mouvement ne relève aucunement de l'évidence.

\section{Que signifie pratiquer l'interdisciplinarité?}

Patrick Charaudeau (2010 : 205) définit ainsi l'interdisciplinarité :

« C'est l'effort d'articuler entre eux les concepts, les outils et les résultats d'analyse de différentes disciplines. Cela ne peut se faire avec plusieurs disciplines à la fois car, pour pouvoir procéder à une interrogation ou un empreint entre plusieurs concepts, il faut pouvoir les considérer dans leur cadre théorique afin de ne pas les déformer, les interroger à la lumière d'une autre discipline et expliquer dans quelle mesure et à quelles fins d'analyse ils peuvent être empruntés et intégrés dans l'autre discipline ».

Pour lui, la notion d'interdisciplinarité caractérise donc autant la démarche consistant à croiser des concepts que celle relative à l'empreint d'outils d'analyse et d'interprétation à d'autres disciplines. II ne peut donc seulement s'agir de la juxtaposition d'approches (pluridisciplinarité), mais de l'association pleine et entière entre plusieurs disciplines. Pour exemplifier son propos, il liste un certain nombre de cas. Relèvent donc de l'interdisciplinarité

\footnotetext{
« le fait que certains courants des sciences du langage intègrent des données sociologiques, sociocommunicatives ou socio-psychologiques dans leur modèle théorique ou dans leur méthodologie; le fait que des psychosociologues du langage intègrent, les uns des éléments de pragmatique $[\ldots]$, les autres d'énonciation [...]; que des chercheurs des sciences de l'information et de la communication s'interrogent sur leur discipline en s'appuyant sur les travaux de sociologues, de sémioticiens et d'analystes du discours [...] à propos de l'analyse des médias, ou que des sociologues se situent à un carrefour entre la science économique et la philosophie politique, et d'autres encore au carrefour de la sociologie, I'histoire ou l'anthropologie » (Charaudeau, $2010: 215)$
}

Roselyne Koren prend résolument parti pour le point de vue défendu par Patrick Charaudeau, précisant qu'elle le fait « contrairement aux lois du genre de la controverse scientifique, $[\ldots]$ et cela non par esprit de corps $[\ldots]$, mais par réelle conviction ». D'ailleurs, son raisonnement atteste de cette conviction 
puisqu'elle montre, à partir d'une étude de cas, les conséquences préjudiciables de l'enfermement monodisciplinaire. Le domaine qui lui sert de laboratoire est celui de la « conversationalisation », un concept commun à des chercheurs en analyse critique du discours, en sciences politiques et en communication numérique. Précisons avant d'aller plus avant que, comme le précise Roselyne Koren, ce " phénomène réfère à ce qui est perçu comme de l' "écrit oralisé", genre que Norman Fairclough [...] qualifie d' "hybride" et qui est apte à introduire directement tout locuteur dans la sphère égalitariste des interactions verbales de la Toile ». Si ce sujet intéresse différentes disciplines, c'est qu'il est à la croisée de plusieurs préoccupations : évidemment discursives, avec une attention portée à l'énonciation du sujet; politiques, d'autant que le sujet qui s'exprime sur la Toile voit son statut et son mode d'adresse se transformer; techniciste, par les lieux et moyens sollicités pour s'exprimer... Mais ce que constate Roselyne Koren, c'est que cet intérêt partagé ne converge pas vers une analyse profitant pleinement des apports de chacune. En effet,

« si elles soulignent toutes les trois les conséquences politiques, culturelles et sociales du phénomène, l'interprétation diverge dans le cas de la détermination des enjeux, qui reste descriptive dans le cas de l'analyse critique du discours et de la communication numérique, mais prend position en termes éthiques dans celui des sciences politiques ».

En cela, elle retrouve ce qu'Edgar Morin (1990) redoutait et qui en appelait, pour y répondre, à une « perestroïka scientifique », c'est-à-dire une démarche osant l'interdisciplinarité, la « multi- ou polydisciplinarité » et la « trans-disciplinarité »:

« L'institution disciplinaire entraîne à la fois un risque d'hyperspécialisation du chercheur et un risque de « chosification » de l'objet étudié dont on risque d'oublier qu'il est extrait ou construit. L'objet de la discipline sera alors perçu comme une chose en soi; les liaisons et solidarité de cet objet avec d'autres objets, traités par d'autres disciplines, seront négligées ainsi que les liaisons et solidarités avec l'univers dont l'objet fait partie. La frontière disciplinaire, son langage et ses concepts propres vont isoler la discipline par rapport aux autres et par rapport aux problèmes qui chevauchent les disciplines. L'esprit hyperdisciplinaire va devenir un esprit de propriétaire qui interdit toute incursion étrangère dans sa parcelle de savoir ».

Si ces chercheurs défendent le principe d'interdisciplinarité, Patrick Charaudeau (2010 : 220) précise quant à lui quelles peuvent être les conditions de réalisation de celle-ci. Il le fait en recourant à la notion d'interdisciplinarité focalisée qu'il définit comme étant « un état d'esprit engendrant une démarche qui cherche à tenir à la fois la multi-appartenance disciplinaire des phénomènes sociaux (interdisciplinarité) et la rigueur d'une discipline (focalisée) ». Roselyne Koren approuve cette option et la peaufine en la rapprochant d'une autre, défendue par Josiane Boutet et Dominique Maingueneau (2005) sous le terme d'« air de famille ». Pourquoi faire appel à cette notion? Pour montrer que l'interdisciplinarité ne doit pas seulement relever d'un rapprochement évident et rationnel, mais d'une forme de ressemblance. Et c'est cette approximation qui, selon Roselyne Koren, est opérante car elle permet d'explorer la complexité des objets. La filiation est ici clairement posée avec Ludwig Wittgenstein (1958) qui, dans le cadre de son étude sur les jeux de langage, rapprochait les mots au vu de leur 
ressemblance familiale avec d'autres, leur « air de famille », c'est-à-dire des traits partagés sans qu'on puisse précisément dire desquels il s'agit. Et de féliciter en conclusion ceux qui, de ses collègues - Josiane Boutet, Dominique Maingueneau, Patrick Charaudeau et Catherine Kerbrat-Orechioni - vont « jusqu'à reconnaître le caractère subjectif du choix des disciplines et des notions qui les inspirent ».

Au-delà de ce jugement qu'elle prononce et revendique, Roselyne Koren considère que l'interdisciplinarité focalisée consiste en une ouverture au sein de l'orthodoxie disciplinaire, à même d'investir des impensés tel, en sciences du langage, celui relatif à ces « actes de parole qui ont l'argumentation de prises de position pour enjeu ». Pourquoi l'analyse des jugements de valeur est-elle un impensé dans cette discipline? « On peut formuler l'hypothèse que cela est dû à un problème scientifique majeur : la rigueur de la construction de l'objet d'une discipline exigerait que les questions d'éthique soient traitées uniquement par ceux qui disposent du cadre théorique et méthodologique adéquat, soient les philosophes ». À cette hypothèse, Roselyne Koren ajoute que « si le vrai et le juste, les jugements de réalité et les jugements de valeur sont également soumis à l'énonciataire par le biais du langage, pourquoi seule la mise en mots du vrai est-elle intégrée dans l'épistémologie des sciences du langage? » Pour pouvoir conduire des travaux sur ces questions, Roselyne Koren a donc transgressé les règles en usage dans sa propre discipline et pratiqué l'interdisciplinarité focalisée, en allant chercher du côté de la rhétorique perelmanienne matière à penser « la prise de position, la décision et l'aspiration à obtenir l'adhésion de l'autre ». Une incursion réussie et profitable dans un ailleurs théorique et méthodologique où deux disciplines sont parvenues à se comprendre pour le bien de l'analyse...

Mais... Qu'est-ce qui fait que des disciplines ne se comprennent pas? Telle est la question que pose Sylvie Leleu-Merviel dans un propos qui prend totalement à rebours celui des autres débatteurs. Pour en comprendre le raisonnement, partons de la conclusion de cette spécialiste des technologies d'information et de communication :

« De ce qui précède, on déduit qu'il existe de nombreuses manières de ne pas se comprendre entre disciplines:

- extraire des fragments différents d'une même réalité sans s'apercevoir que ce ne sont pas les mêmes fragments;

- les examiner sur la base d'aspects qualifiants différents, en ignorant qu'ils sont différents;

- procéder à la qualification par des méthodologiques ou via des outils qui n'ont strictement rien à voir les uns avec les autres;

- prendre pour argent comptant les données des autres alors qu'elles résultent d'un canon descriptionnel totalement inadapté à sa propre approche;

- dissimuler sous un même "étiquetage verbal", un même vocable de "concept", des horizons de pertinence ne partageant rien ».

Pour en arriver à cette mise en garde, Sylvie Leleu-Merviel est partie d'une situation qui ne pouvait être qualifiée d'《 acte de communication », les sujets en interaction se retrouvant dans un cadre médical (un examen à effectuer) où il pas nécessaire d'échanger des paroles. Mais s'il n'y a pas eu à proprement parler 
de communication, des informations, en revanche, ont été transmises, le cas de patient ayant fait l'objet d'une interrogation en vue d'un diagnostic à établir. Pour la chercheuse, cette « historiette » pour le moins ordinaire mettrait à mal le postulat selon lequel,

« lorsqu'il s'agit d'une communication collective qui se déploie dans un espace public à l'aide de divers supports de transmission, on dira que la communication se réalise entre une instance de production du langage, et une instance de réception-interprétation (qui peut devenir à son tour instance de production mais de façon asymétrique), entre lesquels transite un certain produit porteur de sens ayant diverses formes selon le système de signes (verbal, iconique, gestuel) qui le configure » (Charaudeau, 2010:217).

En effet, dans cette petite histoire, qui est l'instance de production? Est-ce le corps défaillant? Le malade lui-même? La machine qui photographie le corps et transmet des informations? Le médecin qui reçoit le patient? Le collectif de spécialistes qui tente de poser un diagnostic? Une interrogation qui conduit Sylvie Leleu-Merviel à constater que Patrick Charaudeau possède son propre horizon de pertinence - une notion réunissant le concept d'horizon d'attente de Hans R. Jauss (1972) et la théorie de la pertinence de Deirdre Wilson et Dan Sperber (1986) -, « celui d'une communication mesurée à l'aune des actes du langage et recourant à l'analyse du discours comme point de vue prioritaire ». Et cet horizon de pertinence a une incidence sur la conception de l'interdisciplinarité qu'il défend. Et de développer, en s'appuyant sur les travaux d'une physicienne, Miora Mugur-Schächter (2009), ce qu'il en est de cet horizon de pertinence quand on change de « lieu géométrique ». On en vient, par exemple, à invalider l'hypothèse d'un « modèle d'analyse de la communication à partir de trois lieux de pertinence séparés : le lieu des conditions de la production, le lieu des conditions de la réception, le lieu des conditions de réalisation de l'acte de communication comme produit fini ».

Plus largement, cela signifie que « le réel en soi » n'existe pas et qu'il faut replacer les phénomènes à observer dans « la relativité de leur perçu », donc dans un cadre où l'expérience occupe une place importante. Montrant que l'expérience est également présente dans le règne animal, Sylvie Leleu-Merviel fait de la créativité une caractéristique humaine. Transposée dans le cadre scientifique et associée à l'expérience, cette compétence signifie que l'activité scientifique qui s'élabore dans un groupe réfère à une dimension intersubjective qui suppose, pour qu'un consensus se manifeste, de trouver une forme de stabilisation à travers des procédures de légalisation. Ce sont elles qui permettront aux membres du groupe d'adhérer à des valeurs communes à même de faire consensus pour qu'elles puissent être communiquées. On voit combien la part des construits est importante, des construits dont il est dit qu'ils sont « façonnés par la méthode de construction, laquelle relève d'un choix cognitif - volontaire et totalement assumé dans la démarche scientifique, souvent réflexe dans la vie courante $\gg$. Au vu de cette démonstration, il apparait qu'un partage de savoir et de connaissances ne peut faire l'impasse sur ces spécificités construites au fil du temps. Ainsi, dans le titre de sa contribution, Sylvie Leleu-Merviel remplace-t- 
elle la notion d'interdisciplinarité par celle d'horizon de pertinence focalisé. Une manière de réintégrer dans les échanges entre chercheurs la complexité de leurs expériences respectives.

\section{Conclusion}

Pour exemplifier l'ensemble des propositions de cette première livraison des «Échanges » sur le thème de l'interdisciplinarité, permettons-nous un détour par un programme interdisciplinaire de recherche dont nous sommes à l'initiative: Qualifier, disqualifier, requalifier des lieux de détention, de concentration et d'extermination'. Réunissant une cinquantaine de chercheurs de différentes nationalités (Allemagne, Argentine, Belgique, France, Canada, Espagne, Pologne, Sénégal, Ukraine), il a pour visée une étude contrastive des lieux ci-dessus cités selon la problématique de la qualification/disqualification/requalification. Dans un premier temps, celle-ci consiste à repérer le geste fondateur - et le sens de celui-ci - par lesquels des acteurs de la mémoire - qu'ils représentent les pouvoirs publics, des citoyens (regroupés ou non en collectifs) ou encore des victimes - font d'un lieu le site emblématique d'une histoire dont ils décident de commémorer un aspect. Dans un deuxième, c'est-à-dire la disqualification, les chercheurs s'attachent à décrire et comprendre les ressorts de la disgrâce au cours de laquelle le lieu peut tomber dans l'oubli, les valeurs qu'il représente pouvant ne pas correspondre aux attentes du moment. Pour la troisième phase, la requalification, il s'agit de prendre en compte les infléchissements de sens du lieu en question, ou bien l'exhumation de lieux oubliés, à la faveur de causes ou situations « nouvelles » qui peuvent, à un moment ou à un autre, surgir dans l'espace public. Les supports servant de terrain aux chercheurs sont évidemment les lieux eux-mêmes, mais pas seulement. II peut s'agir aussi des édifices commémoratifs, des musées, des écrits de victimes...

On est donc confronté à un objet complexe ou dimensions matérielles et immatérielles s'associent, aspects factuel ou imaginaire également. Pour traiter au mieux de cette complexité, les chercheurs viennent donc de cinq disciplines: ethnologie, études littéraires, géographie, histoire, sciences de l'information et de la communication. Sans difficulté, on peut imaginer que Patrick Charaudeau (2010: 199) examinerait ce programme et ses intentions à la lumière de ce qu'il écrit, « mais en même temps le doute s'installe sur la définition de cette

Qualifier, disqualifier, requalifier les lieux de détention, de concentration et d'extermination s'inscrit dans l'axe 4 « Mémoires, culture et sciences » de la MSH Lorraine II a donné lieu à la publication de quatre ouvrages, chacun centré sur une thématique permettant d'étudier les processus à l'œuvre : Qualifier des lieux de détention et de massacre (Fleury, Walter, 2008); Qualifier des lieux de détention et de massacre (2).Territorialisation, déterritorialisation (Fleury, Walter, 2009); Qualifier des lieux de détention et de massacre (3). Figures emblématiques, mobilisations collectives (Fleury, Walter, 20I0); Qualifier des lieux de détention et de massacre (4). Dispositifs de médiation mémorielle (Fleury,Walter, 20II). 
notion lorsque le terme d'interdisciplinarité apparaît en équivalence, parfois en complément avec d'autres termes [...]; à quoi on peut ajouter la concurrence avec d'autres termes comme multidisciplinarité et transdisciplinarité ». Comment lui répondre? Effectivement, si déjà dans la demande de financement (février 2007), ce programme affichait une intention interdisciplinaire, ce n'est qu'au moment de son lancement que cette intention est devenue réalité. En effet, le premier colloque s'est déroulé en novembre 2007, sans que les membres du programme, à quelques exceptions près, ne se soient rencontrés auparavant. Or, c'est en présentant oralement leurs contributions devant des chercheurs d'autres disciplines qu'une expérience commune de recherche a commencé à se constituer et qu'une démarche interdisciplinaire s'est réellement mise en place. Comment? Sur les quatre années d'existence de ce programme, on constate une évolution des façons de faire de chacun qui se manifeste autant dans la construction des objets et le mode d'exploitation les concernant, que dans l'agenda de la recherche. Ainsi sont - évidemment en partie - subsumés certains tropismes disciplinaires. Par exemple, les historiens sont plus attentifs à la question des représentations qu'ils ne l'étaient auparavant, ce qui les conduit à puiser dans la littérature, les revues et/ou la presse matière à penser leurs objets. Les autres chercheurs complètent leurs corpus (ouvrages, presse) d'une investigation plus systématique dans des lieux d'archives. Et si l'on revient sur un point évoqué par Sylvie Leleu-Merviel et qui constituait un frein à l'interdisciplinarité, à savoir l'existence de construits disciplinaires façonnés dans un cadre particulier, nous pensons qu'avoir travaillé ensemble pendant quatre ans a permis à des chercheurs de disciplines différentes de se comprendre et de construire des questionnements et pratiques mêlant des savoirs disciplinaires et d'autres, propres à ce groupe pour ce terrain particulier.

Pour autant, probablement devrions-nous nous méfier des discours enchantés sur l'interdisciplinarité. Et pour méditer jusqu'à la livraison précédente - où deux anthropologues, un philosophe et un historien prendront le relai - voici à lire ce que l'on trouve sur le site du CNRS, à la rubrique « Les programmes interdisciplinaires du CNRS $»^{2}$ :

« Le CNRS est le seul organisme français qui puisse mettre en œuvre des opérations interdisciplinaires innovantes entre ses équipes sur une large palette de disciplines de recherche. Ainsi, les programmes interdisciplinaires du CNRS trouvent leur place dans la structuration des actions de recherche, la définition et l'animation de la politique scientifique et de la stratégie de l'Organisme dans le but d'accompagner les équipes les plus en pointe et les projets les plus porteurs d'avenir, d'assurer la complémentarité entre disciplines voisines et de la pluridisciplinarité d'objectifs.

Les programmes interdisciplinaires du CNRS arrivent en complémentarité avec les programmes de l'ANR et financent des projets à risque aux interfaces couvrant des sujets amont de recherche fondamentale. Ils ouvrent la possibilité de faire émerger des voies nouvelles pouvant présenter une prise de risque. En outre, les programmes interdisciplinaires se démarquent en permettant de promouvoir des démarches nouvelles innovantes et originales.

\footnotetext{
${ }^{2}$ Accès : http ://www.cnrs.fr/prg/PIR/presentation.htm. Consulté en juillet 2010.
} 
Les objectifs majeurs des Programmes Interdisciplinaires se déclinent dans diverses actions:

- Susciter l'émergence de nouvelles thématiques à la frontière des différentes structures traditionnelles;

- Répondre à des défis scientifiques et technologiques ainsi qu'à des enjeux socioéconomiques ou à des problèmes de société;

- Structurer la communauté scientifique en favorisant les échanges entre chercheurs de disciplines différentes ou en facilitant la mise en place de structures d'accueil et d'équipements mutualisés dans les laboratoires;

- Favoriser la construction de dossiers solides et d'organisations structurées qui seront proposés ensuite à l'ANR, ou aux appels d'offres européens... ».

\section{Références}

Berthelot J.-M., 1996, Les vertus de l'incertitude, Paris, Presses universitaires de France.

Boure R., 2006, « L'histoire des sciences de l'information et de la communication ( I ). Entre gratuité et réflexivité », Questions de communication, 10, pp. 277-295.

— 2007, « L'histoire des sciences de l'information et de la communication (2). Le cas des origines littéraires des SIC », Questions de communication, I I , pp. 257-287.

- 2008, « L'histoire des sciences de l'information et de la communication (3). Postures, concepts et méthodes en débat 》, Questions de communication, |3, pp. | 3 | - 152.

Boutet J., Maingueneau D., 2005, « Présentation », Langage \& société, I | 4, pp. 9- 13.

Boutier J., Passeron J.-Cl., Revel J., éds, 2006, Qu'est-ce qu'une discipline?, Paris, Éd. de l'École des hautes études en sciences sociales.

Charaudeau P., 2010, « Pour une interdisciplinarité "focalisée" dans les sciences humaines et sociales », Questions de communication, 17, pp. 195-222.

Fleury B.,Walter J., 2007, « L'histoire des sciences de l'information et de la communication », Questions de communication, 12, pp. 133-148.

- 2008, dirs, Qualifier des lieux de détention et de massacre, Nancy, Presses universitaires de Nancy.

- 2009, dirs, Qualifier des lieux de détention et de massacre (2). Territorialisation, déterritorialisation, Nancy, Presses universitaires de Nancy.

- 2010, dirs, Qualifier des lieux de détention et de massacre (3). Figures emblématiques, mobilisations collectives, Nancy, Presses universitaires de Nancy.

- 2011, dirs, Qualifier des lieux de détention et de massacre (4). Dispositifs de médiation mémorielle, Nancy, Presses universitaires de Nancy.

Jauss H. R., 1972, Pour une esthétique de la réception, trad. de l'allemand par Cl. Maillard, Paris, Gallimard, 1978.

Morin E., 1990, « Sur l'interdisciplinarité », pp. 21-31, in: Kourilsky F., dir., Carrefour des sciences, Paris, Éd. cNRS. Accès : http ://basarab.nicolescu.perso.sfr.fr/ciret/bulletin/b2c2. htm. Consulté en juillet 2010.

Mugur-Schächter M., 2009, L'infra-mécanique quantique. Une révolution épistémologique révélée dans les descriptions de microétats, Paris, Éd. Dianoïa. 
Interdisciplinarité, interdisciplinarités

Olivesi S., 2007, « À propos de l'institutionnalisation des sıc. Pour une histoire "focalisée" », Questions de communication, 12, pp. 203-225.

Questions de communication, 2004a, « Des usages du constructivisme », 5, pp. |6 |-2। |.

— 2004b, « Des usages du constructivisme (2) 》, 6, pp. I 01 - I 77.

— 2005a, «Terrain, expérimentation et sciences sociales », 7, pp. |47-232.

— 2005b, «Terrain, expérimentation et sciences sociales (2) », 8, pp. I | 3- 64.

— 2007, « L'histoire des sciences de l'information et de la communication », I2, pp. I33226.

- 2009a, «Penser le genre en sciences de l'information et de la communication et au-delà », | 5, pp. | | |-260.

- 2009b, «Penser le genre en sciences de l'information et de la communication et au-delà (2) », |6, pp. |3|-209.

Wilson D., Sperber D., 1986, La pertinence. Communication et cognition, trad. de l'anglais par A. Gerschenfeld et D. Sperber, Paris, Éd. de Minuit, 1989.

Wittgenstein L., 1958, Le cahier bleu ou le cahier brun, trad. de l'allemand par M. Goldberg et J. Sackur, Paris, Gallimard, 1996. 
$-1$

$1_{-}^{1}$

$-1$

$1^{-}$ 